\title{
Erratum to: Coarse-grained model of the native cellulose $I \alpha$ and the transformation pathways to the $I \beta$ allomorph
}

\author{
Adolfo B. Poma - Mateusz Chwastyk • \\ Marek Cieplak
}

Published online: 9 April 2016

(C) Springer Science+Business Media Dordrecht 2016

\section{Erratum to: Cellulose \\ DOI 10.1007/s10570-016-0903-4}

In the Original publication of the article, the acknowledgment missed one of the funding agencies. The correct acknowledgement should be:

This research has been supported by the ERA-NETIB/06/2013 Grant FiberFuel funded by the National
Centre for Research and Development in Poland, the ERA-NET grant ERA-IB (EIB.12.022) (FiberFuel) and the European Framework Programme VII NMP grant 604530-2 (CellulosomePlus). It was also cofinanced by the Polish Ministry of Science and Higher Education from the resources granted for the years 2014-2017 in support of international scientific projects.
A. B. Poma $(\varangle) \cdot$ M. Chwastyk · M. Cieplak Institute of Physics, Polish Academy of Sciences, Aleja Lotników 32/46, 02-668 Warsaw, Poland e-mail: poma@ifpan.edu.pl 